\title{
Pharmaceuticals residues in selected tropical surface water bodies from Selangor (Malaysia): occurrence and potential risk assessments
}

\begin{abstract}
This study investigated the occurrence of nine pharmaceuticals (amoxicillin, caffeine, chloramphenicol, ciprofloxacin, dexamethasone, diclofenac, nitrofurazone, sulfamethoxazole, and triclosan) and to evaluate potential risks (human health and ecotoxicological) in Lui, Gombak and Selangor (Malaysia) rivers using commercial competitive Enzyme-Linked Immunosorbent Assay (ELISA) kit assays. Physicochemical properties of these rivers showed the surface samples belong to Class II of Malaysian National Water Quality Standards which requires conventional treatment before consumption. All the pharmaceuticals were detected in all three rivers except for triclosan, dexamethasone and diclofenac which were not detected in few of sampling locations in these three rivers. Highest pharmaceutical concentrations were detected in Gombak river in line of being as one of the most polluted rivers in Malaysia. Ciprofloxacin concentrations were detected in all the sampling locations with the highest at $299.88 \mathrm{ng} / \mathrm{L}$. While triclosan, dexamethasone and diclofenac concentrations were not detected in a few of sampling locations in these three rivers. All these nine pharmaceuticals were within the levels reported previously in literature. Pharmaceutical production, wastewater treatment technologies and treated sewage effluent were found as the potential sources which can be related with pharmaceuticals occurrence in surface water samples. Potential human risk assessment showed low health risk except for ciprofloxacin and dexamethasone. Instead, ecotoxicological risk assessment indicated moderate risks were present for these rivers. Nevertheless, results confirmation using instrumental techniques is needed for higher degree of specificity. It is crucial to continuously monitor the surface water bodies for pharmaceuticals using a cost-effective prioritisation approach to assess sensitive sub-populations risk.
\end{abstract}

Keyword: Pharmaceuticals; Surface water; ELISA; Occurrence; Potential risks 